\title{
Hybridoma technology: the preferred method for monoclonal antibody generation for in vivo applications
}

\author{
Samantha Zaroff ${ }^{\star, 1}$ \& Grace Tan'
}

$\mathrm{P}$ rior to the development of hybridoma technology, researchers relied on polyclonal antibodies (pAbs) for experiments involving the identification and quantification of specific proteins of interest within complex biological environments. While pAbs offered their own set of benefits, they could not be used for in vivo experiments or therapeutics due to their batch-to-batch inconsistency and high levels of background reactivity. It was not until 1975 when Köhler and Milstein used hybridomas to generate monoclonal antibodies (mAbs), that the worlds of antibodies and in vivo scientific research could meet for the first time [1,2]. The introduction of mAbs to in vivo research gave rise to some of the greatest scientific achievements of the 21 st century, including in vivo diagnostics, mAb therapeutics and antibody-drug conjugation. While other technologies such as display libraries have since been developed for mAb discovery, hybridoma technology remains the primary discovery platform preferred by in vivo scientists. This editorial will address the benefits of using hybridoma technology for in vivo applications and describe some of the major contributions that hybridomaderived mAbs have made in in vivo research and therapy.

\section{WHAT IS HYBRIDOMA TECHNOLOGY?}

Hybridomas are cells formed via fusion between a short-lived antibody-producing B cell and an immortal myeloma cell. Each hybridoma constitutively expresses a large

KEYWORDS

antibodies $\cdot$ cancer $\cdot$ immunology $\cdot$ immunotherapy $\bullet$ in vitro models $\cdot$ reagents

${ }^{1}$ GenScript USA, Piscataway, NJ 08854, USA; *Author for correspondence: samantha.zaroff@genscript.com

BioTechniques 67: $90-92$ (September 2019) 10.2144/ btn-2019-0054 amount of one specific mAb, and favored hybridoma cell lines can be cryopreserved for long-lasting $\mathrm{mAb}$ production. As a result, researchers usually prefer generating hybridomas over other mAb production methods in order to maintain a convenient, never-ending supply of important mAbs.

Hybridoma generation is a five-step process that takes advantage of a host animal's natural ability to generate functional, highly specific, high-affinity mAbs. In brief, the first stage involves the development and optimization of an immunogenic antigen (Ag) [3]. Next, a imaging. Some common imaging methods include positron emission tomography (PET), magnetic resonance imaging (MRI), fluorescent molecular tomography (FMT) and ultrasound $[6,7]$. The main difference between immune imaging and standard imaging is that, rather than imaging a large, nonspecific section of the body, a tagged antibody targets a precise location for diagnostic imaging instead. This idea of conjugating a full-length antibody or an antibody fragment to a nanoparticle, be it a radioisotope, fluorophore or positron emitter, would have not been possible without hybridoma-based antibody

\section{With ADCs and targeted biologics on the rise, there is no telling what astounding discovery will revolutionize mAb therapeutics next.99}

host animal is immunized with the Ag to elicit an immune response and initiate the process of B-cell maturation [2,4]. The third stage involves the isolation of these $B$ cells from the spleen of the host animal and their fusion with myeloma cells to generate hybridomas [5]. During the fourth stage, the generated hybridomas are subject to multiple rounds of screening and selection in order to identify the hybridomas that produce the best mAbs for the intended downstream application. The fifth and final stage is the amplification of these specific hybridomas and subsequent $\mathrm{mAb}$ purification [3].

\section{COMMON IN VIVO APPLICATION OF mAbS In vivo diagnostics}

In vivo diagnostics are a noninvasive way for clinicians to diagnose disease progression through analysis of biomarkers within the body rather than through biologic samples inside a laboratory. Most antibody-based in vivo diagnostics are used for highly specific discovery. It was only through hybridoma technology that fully natural mAb variable domains that did not adversely impact a patient's own immune system during an examination were discovered.

\section{mAb therapeutics}

Compared with other biologics, mAbs are able to maintain an extremely high affinity towards their target. Due to this high affinity and specificity, researchers began investigating the therapeutic potential of mAbs as metabolic activators, inhibitors and immunomodulators. While the first few US FDA-approved mAb therapeutics, such as muromonab-CD3, were generated solely in mice, it became evident that in order to avoid immune rejection, future $m A b$-based therapeutics needed to undergo humanization [8-10]. Since the approval of muromonab-CD3 in 1986, the FDA has approved approximately 80 more mAb therapeutics for diseases ranging from autoimmune disorders, to inflammatory diseases, HIV and cancer. Interestingly, > 
- despite the discovery of combinatorial display libraries in 1984 as an alternative $\mathrm{mAb}$ discovery platform, the majority of these $m A b$ therapeutics were originally discovered using hybridoma technology in either fully murine or humanized mice. The reason for this preference is likely attributed to the natural ability of the murine immune system to generate highly specific mAbs that elicit strong constant domain functionality with limited immunoreactivity after humanization.

\section{Antibody-drug conjugates}

\& targeted biologics

Like in vivo diagnostics, mAbs can be conjugated to almost any small molecule, be it a fluorophore or a drug, in order to deliver cargo to a specific target. Targeted therapeutics come in two flavors, antibody-drug conjugates (ADCs) and targeted biologics [11]. ADCs are formed when a mAb or $\mathrm{mAb}$ fragment is conjugated to a smallmolecule drug for targeted delivery to a specific organ, tissue or tumor. These "magic bullets" have revolutionized the field of targeted drug delivery, especially for oncology and immunology. After the success of ADCs, researchers took the idea one step further and discovered a way of expressing antibody variable chains on the surface of living drugs, or biologics. One of the most popular forms of targeted biologics is chimeric antigen receptor T-cell therapy (CAR-T) [12]. Unlike ADCs, CAR-T targets a $T$ cell to a tumor-associated antigen (TAA) through single-chain variable fragment (ScFvs) recombinantly expressed on the T-cell surface. Once the ScFv interacts with the TAA, the T cell becomes activated and initiates an immune response directly targeting the tumor. Novel forms of targeted biologics are being discovered every day, such as bispecific CARs and T-cell receptor therapy [13]. With ADCs and targeted biologics on the rise, there is no telling what astounding discovery will revolutionize $\mathrm{mAb}$ therapeutics next.

\section{BENEFITS OF HYBRIDOMA TECHNOLOGY FOR IN VIVO APPLICATIONS}

Natural affinity maturation

Since hybridoma technology relies on mature B cells taken from secondary lymphatic organs, purified mAbs from these cells will have already gone through multiple stages of stringent genetic modifications within the host animal. Natural affinity maturation (AM) is a complex antibody refinement process that occurs within the germinal centers of the host animal [14]. During AM, DNA encoding the variable regions of antibody chains are diversified via somatic hypermutation (SHM) $[15,16]$. Through SHM, mutations accumulate in the complementary-determining regions (CDRs) of the variable genes, and the tightest binders to the Ag are actively selected for, resulting in the production of antibodies with high affinity. In addition to SHM, several mammalian species (rabbits) and birds also undergo gene conversion (GCV). GCV takes place when a dsDNA break occurs within the already rearranged $V(D) J$ segments of an

\section{Somatic hypermutation and gene conversion improve antibody affinity within the variable region; however, the constant domain of the antibody also has to go through its own maturation process. ,'}

antibody's variable region and the break is repaired via a homologous recombinationlike mechanism, introducing affinityincreasing mutations in the process.

\section{Low level of immunogenicity}

Since hybridoma technology depends on the immunization of a host animal, the generated high-affinity antibodies will possess natural pairing of variable light $\left(\mathrm{V}_{\mathrm{L}}\right)$ and heavy $\left(\mathrm{V}_{\mathrm{H}}\right)$ gene segments with a class-matched constant domain. During the process of humanization, all regions surrounding the CDRs are modified in order to reduce the chance of the antibody causing a negative immune response once inside a patient. Since the process of hybridoma-based $\mathrm{mAb}$ generation takes advantage of natural CDR development, there is very limited risk of those CDRs causing an immune response. In vitro methods, such as display libraries, can generate unnatural pairings of $V_{H}$ and $V_{\perp}$ and cause the resulting $m A b$ to have high immunogenicity, especially after unnatural $\mathrm{AM}$ and recombinant pairing with a standard IgG backbone [17]

\section{Constant domain activity}

SHM and GCV improve antibody affinity within the variable region; however, the constant domain of the antibody also has to go through its own maturation process. Newly generated antibodies will generally be of the lgM isotype. In order to change their isotype, antibodies will go through class switch recombination (CSR), a process that facilitates isotype switching [18]. IgG is the most common isotype used in biological research and drug development; however, CSR can generate antibodies from all five isotypes in order to ensure that the antibody is equipped with the necessary effector functions and different bio-distribution

\section{SUMMARY}

This editorial reviews the basics of hybridoma technology, the common uses of mAbs in in vivo applications, and the technical benefits of using hybridomas to generate $m A$ Abs for these applications. Alternative methods for mAb discovery and development, such as in vitro combinatorial display libraries and in vivo B-cell sorting, have also been successfully developed $[17,19,20]$. Display libraries rely on screening large libraries of randomized, synthetic variable $V_{H}$ and $V_{L}$ antibody fragments in order to select the tightest $\mathrm{Ag}$ binders, while $B$-cell sorting relies on identifying and sorting mature B cells from peripheral blood. Unlike hybridoma technology, these methods rely heavily on recombinant $\mathrm{mAb}$ expression as the basis of mAb production. While each method certainly has their respective merits and drawbacks, hybridoma technology, despite being a "senior" antibody generation methodology in 2019 , still prevails as the most preferred method for discovering mAbs for in vivo applications. With new discoveries made every day to enhance antibody discovery, 
the future looks very bright for adapting hybridoma technology to support a wide range of in vivo applications.

\section{FINANCIAL \& COMPETING INTERESTS DISCLOSURE}

This article was funded in its entirety by GenScript USA global life science contract research organization. The authors have no other relevant affiliations or financial involvement with any organization or entity with a financial interest in or financial conflict with the subject matter or materials discussed in the manuscript apart from those disclosed.

No writing assistance was utilized in the production of this manuscript.

\section{OPEN ACCESS}

This work is licensed under the AttributionNonCommercial-NoDerivatives 4.0 Unported License. To view a copy of this license, visit http://creativecommons.org/licenses/ by-nc-nd/4.0/

\section{REFERENCES}

1. Kohler G, Milstein C. Continuous cultures of fused cells secreting antibody of predefined specificity. Nature 256(5517), 495-497 (1975)

2. De Almeida R, Nakamura CN, De Lima Fontes $\mathrm{M}$ et al. Enhanced immunization techniques to obtain highly specific monoclonal antibodies. MAbs 10(1), 46-54 (2018).

3. Council NR. Monoclonal Antibody Production. National Academies Press, Washington, DC,
USA, doi:10.17226/9450 (1 999).

4. Yatim KM, Lakkis FG. A brief journey through the immune system. Clin. J. Am. So. Nephrol. 10(7), 1274-1281 (2015).

5. Karsten U, Stolley P, Walther I et al. Direct comparison of electric field-mediated and PEG-mediated cell fusion for the generation of antibody producing hybridomas. Hybridoma 7(6), 627-633 (1988).

6. Bannas P, Lenz A, Kunick $V$ et al. Validation of nanobody and antibody based in vivo tumor xenograft NIRF-imaging experiments in mice using ex vivo flow cytomety and microscopy. J. Vis. Exp. 98, e52462 (2015)

7. Sohn $\mathrm{CH}$, Park SP, Choi SH et al. MRI molecular imaging using GLUT1 antibody- $\mathrm{Fe}_{3} \mathrm{O}_{4}$ nanoparticles in the hemangioma animal model for differentiating infantile hemangioma from vascular malformation. Nanomedicine 11(1), 127-135 (2015)

8. Smith SL. Ten years of orthoclone OKT3 (muromonab-CD3): a review. J. Transpl. Coord. 6(3), 109-119; quiz 120-101 (1996).

9. Nelson AL, Dhimolea E, Reichert JM. Development rends for human monoclonal antibody therapeutics. Nat. Rev. Drug Discov. 9(10), 767-774 (2010).

10. Grilo AL, Mantalaris A. The increasingly human and profitable monoclonal antibody market. Trends Biotechnol. 37(1), 9-16 (2019)

11. Shen W-C. Antibody-drug conjugates: a historical review. In: Antibody-Drug Conjugates: The 21st Century
Magic Bullets for Cancer. Wang J, Shen W-C, Zaro JL (Eds). Springer International Publishing Cham. 3-7 (2015)

12. June $\mathrm{CH}$, O'connor RS, Kawalekar OU, Ghassemi $\mathrm{S}, \mathrm{Mi}-$ lone MC. CAR T cell immunotherapy for human cance Science 359(6382), 1361-1365 (2018).

13. Tang $L$, Zheng $Y$, Melo $M B$ et al. Enhancing T cell therapy through TCR-signaling-responsive nanoparticle drug delivery. Nat. Biotechnol. 36(8), 707-716 (2018).

14. Nutt SL, Hodgkin PD, Tarlinton DM, Corcoran LM. The generation of antibody-secreting plasma cells. Nat. Rev. Immunol. 15(3), 160-171 (2015).

15. Di Noia JM, Neuberger MS. Molecular mechanisms of antibody somatic hypermutation. Annu. Rev. Biochem. $76,1-22$ (2007).

16. Peled JU, Kuang FL, Iglesias-Ussel MD et al. The biochemistry of somatic hypermutation. Annu. Rev. Immunol. 26, 481-511 (2008)

17. Steinwand M, Droste P, Frenzel A, Hust M, Dubel S, Schirrmann T. The influence of antibody fragment format on phage display based affinity maturation of $\mathrm{IgG}$. MAbs 6(1), 204-218 (2014).

18. Stavnezer J, Guikema JE, Schrader CE. Mechanism an regulation of class switch recombination. Annu. Rev. Immunol. 26, 261-292 (2008).

19. Bradbury AR, Sidhu S, Dubel S, Mccafferty J. Beyond natural antibodies: the power of in vitro display technologies. Nat. Biotechnol. 29(3), 245-254 (2011).

20. Hawkins RE, Russell SJ, Winter G. Selection of phage antibodies by binding affinity. Mimicking affinity

\section{The ONLY way \\ to accurately Repeat Pipette using Serological Pipets!}

Pipetting is FASTER with ali- $\mathbf{Q}^{\mathrm{m}}$

- Faster and easier multidispensing with NO NEED to watch the lines.

- Repeatable accuracy* with the press of one button!

- Plus aspirate and dispense as you're used to.

- Use any size or brand of serological pipets.

- It's a 2-in-1, regular \& repeating, pipet controller!

Ideal for cell culture, dispensing media, filling plates, tubes, cryovials, etc.

"This product saves us a great deal of time when performing large aliquotting

jobs and does it more accurately than the average bench tech." - For more reviews see www.selectscience.net

${ }^{*}$ get $2 \%$ accuracy and $2 \% \mathrm{CV}$ using Wobble-not ${ }^{\mathrm{TM}}$ serological pipets. See www.vistalab.com/ali-q-pipet-controller for more info and specs.
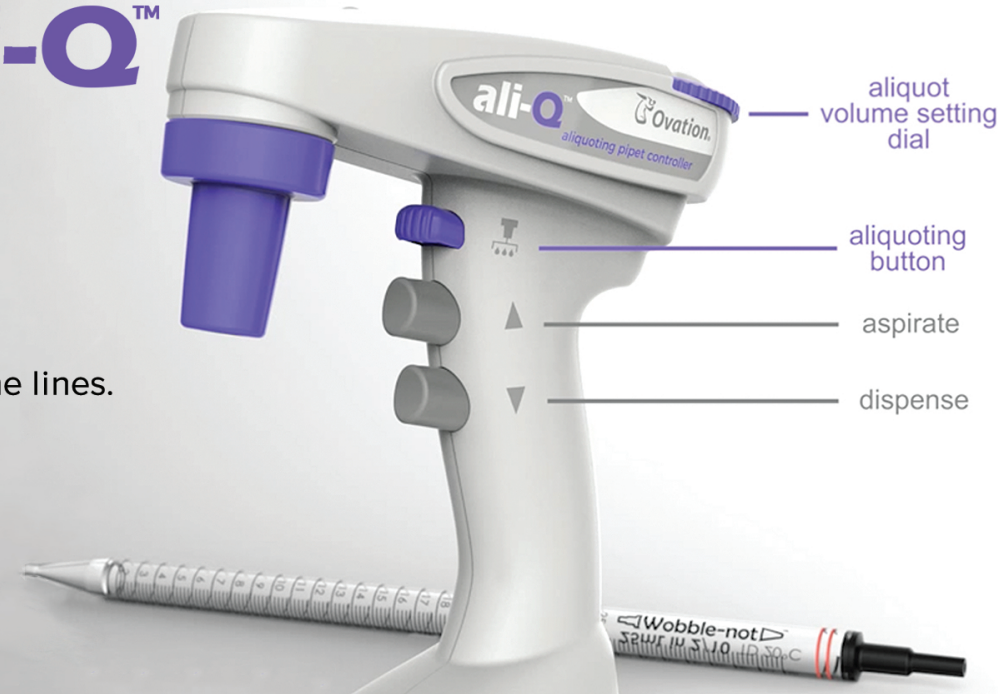

$\sqrt{\text { istalab }}$ Think Differently. Feel Better.

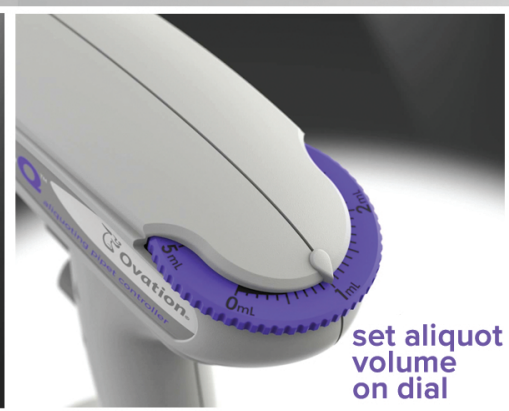
volume
on dial

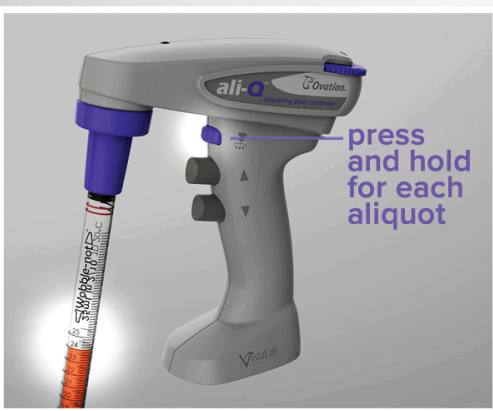

